\title{
Risk attitudes and informal employment in a developing economy
}

\author{
John Bennett ${ }^{1,2^{*}}$, Matthew Gould ${ }^{3}$ and Matthew D Rablen ${ }^{1,2}$
}

*Correspondence:

john.bennett@brunel.ac.uk

${ }^{1}$ Department of Economics and

Finance, and CEDI, Brunel University,

Uxbridge, Middlesex, UB8 3PH, UK

${ }^{2}$ IZA, Bonn, Germany

Full list of author information is

available at the end of the article

\begin{abstract}
We model an urban labour market in a developing economy, incorporating workers' risk attitudes. Trade-offs between risk aversion and ability determine worker allocation across formal and informal wage employment, and voluntary and involuntary self employment. Greater risk of informal wage non-payment can raise or lower informal wage employment, depending on the source of risk. Informal wage employment can be reduced by increasing detection efforts or by strengthening contract enforcement for informal wage payment. As the average ability of workers rises, informal wage employment first rises, then falls. Greater demand for formal production may lead to more involuntary self employment.
\end{abstract}

JEL Classification: 017, J23, D81

Keywords: Risk attitudes, Informality, Self employment

\section{Introduction}

In developing economies the majority of urban employment is informal (OECD 2009), and whether as wage- or self-employment, informality can involve significant risks for the worker. Informal workers have little social protection and relatively volatile earnings, while microfirms have a high rate of failure (Perry et al. 2007; Günther and Launov 2012). An absence of legal protection by the police or courts may leave workers at risk of being cheated (Deshingkar and Akter 2009). Lack of application of health and safety regulations may expose both informal workers and the self employed to significant health risks, and the growth of informality is associated with a greater incidence of depression and stressrelated illnesses (World Bank 2012).

However, in virtually all of the theoretical literature on labour market informality in developing economies, workers are either assumed to be risk-neutral or risk is disregarded altogether. In the seminal paper by Rauch (1991), which is an adaptation of the Lucas (1978) formulation of firm size-distribution to informal labour markets, the focus is on the heterogeneity of agents' (entrepreneurial) ability, but there is no allowance for risk aversion. ${ }^{1}$ In the informality literature based on search-and-matching theory the risk of not being able to find a job is a central feature, but workers are assumed risk neutral (see, e.g., Zenou 2008; Albrecht et al. 2009).

\section{Springer}

(c) 2012 Bennett et al.; licensee Springer. This is an Open Access article distributed under the terms of the Creative Commons Attribution License (http://creativecommons.org/licenses/by/2.0), which permits unrestricted use, distribution, and reproduction in any medium, provided the original work is properly cited. 
The present paper contributes to the Lucas-Rauch branch of the literature by introducing risk aversion. We allow for uncertainty of earnings and we assume that workers, who are heterogeneous, are characterized in two dimensions - risk aversion and ability. In our model, a worker can be in one of three labour market states - formal wage employment, informal wage employment or self-employment. We assume that formal wage employment gives a certain income, but the other two states generate uncertain income, with the uncertainty being greater (in a sense that we specify) for self-employment than for informal wage employment. ${ }^{2}$ The formal wage is assumed to exceed the maximum possible level of the informal wage. Ability is assumed to affect earnings in self-employment, but not in either type of wage employment. We take the demand side of the labour market as given, with downward-sloping demand functions for each type of labour, and we focus on the supply of labour to the three types of work.

The great majority of urban self-employment in developing economies can be classified as informal. In Latin America and the Caribbean, for example, according to Perry et al. (2007), 26.58\% of urban workers are self-employed (Perry et al. use the term 'independent'), of whom $23.64 \%$ are 'informal' and only $2.94 \%$ are 'professionals'. We may therefore regard all self employment (or at least involuntary self employment) as informal in our model. ${ }^{3}$ As specified by Rauch (1991) and much of the ensuing literature on informality, we assume that the wage rate in the formal sector is fixed above the marketclearing level. This might be because of a minimum wage law or union activity; it might be an implicit representation of social security benefit provision; or it might be because formal firms are relatively large, so that monitoring of workers is difficult and employers choose to pay an efficiency wage to deal with moral hazard.

The model is formulated in Section 2. Workers may differ with respect to risk aversion and ability, though each has constant relative risk aversion. Each individual worker may, in principle, gain formal wage employment, informal wage employment or self employment. Because the latter two types of employment yield uncertain income, we specify von Neumann-Morgenstern utility. Because the formal wage rate is set above the marketclearing level, there is rationing of formal jobs and so not all workers who prefer formal employment will achieve it. For each worker we specify the first preference among the three employment states as a function of risk attitude and ability. Under our assumptions informal wage work can never be a first preference. However, formal wage jobs are rationed, and so some of the workers whose first preference is formal wage employment are forced to turn to other work. For these workers we specify a second preference, which can only be for self employment or informal wage employment. Again, we specify this choice as a function of risk attitude and ability. Self employment here is defined as 'involuntary', whereas self-employment as a first choice is defined as 'voluntary." Worker preferences across risk attitude and ability characteristics are illustrated diagrammatically.

In Section 3, assuming that the informal wage rate is market-clearing, we specify conditions for the existence of equilibrium and we illustrate the equilibrium by reinterpreting the diagram formulated in Section 2. We then derive and discuss the comparative statics of the model, examining the effects on the labour market of several policy changes and of some simple representations of economic development. Section 4 concludes, and proofs are given in an appendix. 


\section{The model}

\subsection{Employment}

We consider a population of workers, each of whom is characterized in terms of two parameters, relative risk aversion $\gamma \in[0,1)$ and ability $\rho \in[1, \bar{\rho}]$. Each has a utility function of the form ${ }^{5}$

$$
u(y)=\frac{y^{1-\gamma}}{1-\gamma},
$$

where $y$ denotes income. There are three types of employment: formal wage employment, informal wage employment and self-employment, which are denoted by the respective subscripts $E=(F, I, S)$. A worker cannot engage in more than one type of employment simultaneously. Income $y$ is assumed to be certain in formal wage employment, but uncertain in informal wage employment and self-employment. Ability $\rho$ is assumed only to affect income $y$ in self-employment.

If a worker is employed in a formal job, income $y=w_{F}>0$ with certainty. If employed informally, $y=w_{I}$, where $0<w_{I}<w_{F}$, with probability $1-\phi$, and zero with probability $\phi$, where $\phi \in(0,1)$. The potential zero income is a normalization to simplify the exposition. However, if a worker is self employed, ability $\rho$ may affect income. Income $y$ is then $\rho$ with probability $1-\psi$, and zero with probability $\psi$, where $\psi \in(0,1)$. We can interpret ability $\rho$ as the number of efficiency units of labour that a worker would supply if self-employed. This implicitly sets the wage rate for self-employment as the numéraire., and it implies that $w_{F}$ and $w_{I}$ are wage rates relative to the implicit self-employment wage rate. Von Neumann-Morgenstern utility $v_{E}(E=F, I, S)$ in each of the three types of employment is therefore

$$
v_{F}=u\left(w_{F}\right) ; \quad v_{I}=(1-\phi) u\left(w_{I}\right) ; \quad v_{S}=(1-\psi) u(\rho) .
$$

To capture the idea that self-employment is riskier than informal wage employment, we consider a person whose ability $\rho$ is such that mean income is the same for $S$ as for $I:(1-\phi) w_{I}=(1-\psi) \rho$. Writing $\operatorname{Var}_{E}$ for the variance of income in employment $E=$ $(F, I, S)$, we have

$$
\operatorname{Var}_{I}=\phi w_{I}^{2}(1-\phi) ; \quad \operatorname{Var}_{S}=\rho^{2} \psi(1-\psi) .
$$

Therefore, if $(1-\phi) w_{I}=(1-\psi) \rho$, then $\operatorname{Var}_{S}>\operatorname{Var}_{I}$ if and only if $\psi /(1-\psi)>$ $\phi /(1-\phi)$, which holds if and only if $\psi>\phi$. We shall assume this condition to hold throughout.

\subsection{Labour demand}

Labour demand is formulated as simply as possible, with linear downward sloping curves. Since the implicit wage rate for self-employed labour is the numéraire, we need only formulate behaviour in the formal and informal wage labour markets; by Walras' Law, this will also give us equilibrium in the market for self-employed labour. We therefore only consider labour demand in the two wage labour markets. It is assumed that employers are risk-neutral.

In the informal wage labour market, labour demand will depend on the form of the uncertainty that obtains. Suppose first that the 'discovery' that a firm is employing workers informally leads to the confiscation of all revenue and the nonpayment of any wages with probability $\phi_{c}$. Then marginal cost and marginal revenue are both reduced for the 
employer by the proportion $\phi_{c}$, so that the risk of discovery has no effect on labour demand. Alternatively, suppose the risk for the worker is that the employer will disappear without paying any wages. If the probability of this happening is $\phi_{d}$, the effective wage rate for the employer is $\left(1-\phi_{d}\right) w_{I}$. By writing $\phi_{c}+\phi_{d}=\phi$ we accommodate both of these types of uncertainty.

Denoting the aggregate demand for labour of employment type $E$ by $L_{E}^{d}(E=F, I, S)$, we can therefore write

$$
\begin{aligned}
& L_{F}^{d}=A_{F}-w_{F} ; \\
& L_{I}^{d}=A_{I}-\left(1-\phi_{d}\right) w_{I} ;
\end{aligned}
$$

where $A_{F}$ and $A_{I}$ are positive constants.

\subsection{Labour supply}

We shall assume that the formal wage rate $w_{F}$ is fixed above the market-clearing level. As a result, there will be rationing of formal wage jobs. First we characterize workers' first preferences among $E=F, I, S$. Then, for workers whose first preference is $E=F$, and who may therefore find that they cannot achieve their first preference, we consider second preferences.

\subsubsection{First preferences}

We wish to determine the first preference among $E=F, I, S$ for any worker with characteristics $(\gamma, \rho)$. The first choice sets are given by

$$
\begin{aligned}
& W_{F}: v_{F}>\max \left(v_{I}, v_{S}\right) ; \\
& W_{I}: v_{I}>\max \left(v_{F}, v_{S}\right) \\
& W_{S}: v_{S}>\max \left(v_{F}, v_{I}\right) ;
\end{aligned}
$$

where $W_{E}$ denotes the set of workers whose first preference is $E(E=F, I, S)$.

As $w_{F}>w_{I}, \phi \geq 0$ and $\gamma \in[0,1)$, it follows that $v_{F}>v_{I}$, and hence $W_{I}=\varnothing: E=$ $I$ can never be a first preference. Using (2), we can determine the borderline values of parameters underlying (5) and (7):

$$
v_{F} \gtrless v_{S} \Leftrightarrow \alpha \lessgtr g(\gamma) \text {, }
$$

where $\alpha \equiv \log \rho$ and

$$
g(\gamma)=\log w_{F}-\frac{\log (1-\psi)}{1-\gamma} .
$$

Formal employment is preferred to self employment by workers with a combination of a sufficiently low level of ability and a sufficiently great level of risk aversion. Note that $g(\gamma)$ may take either sign.

We assume a bivariate uniform distribution for log ability, $\alpha$, and risk aversion, $\gamma$, across workers. Since $\rho \in[1, \bar{\rho}]$, we have that $\alpha \in[0, \bar{\alpha}]$, where $\bar{\alpha} \equiv \log \bar{\rho}$. Given that $\gamma \in[0,1)$, the probability density function is

$$
f(\alpha, \gamma)=\frac{1}{\bar{\alpha}} \text {. }
$$


The proportions of workers whose first preferences are formal wage employment and self employment are, respectively,

$$
\begin{aligned}
& \left|W_{S}\right|=\int_{0}^{g^{-1}(\bar{\alpha})} \int_{\max (0, g(\gamma))}^{\bar{\alpha}} f(\alpha, \gamma) \mathrm{d} \alpha \mathrm{d} \gamma \\
& \left|W_{F}\right|=1-\left|W_{S}\right|
\end{aligned}
$$

The expression for $\left|W_{S}\right|$ gives the proportion preferring self employment over levels of risk aversion $\gamma$ up to the critical level $g^{-1}(\bar{\alpha})$ and for log ability at or above the critical level $g(\gamma)$. The remaining portion of the population of workers are in set $W_{F}$, preferring formal wage employment.

These preferences are illustrated in Figure 1, where $\log$ ability $\alpha$ is measured on the horizontal axis and risk aversion $\gamma$ on the vertical axis. The borderline combinations of $(\alpha, \gamma)$ at which a worker is indifferent between formal wage employment and self employment are shown by the upward-sloping line. ${ }^{6}$ Self-employment is preferred by workers with a combination of relatively low risk aversion and relatively high ability. Starting at a borderline combination of $(\alpha, \gamma)$, an incremental increase in risk aversion makes a worker prefer formal wage employment, but a large enough increase in log ability brings the worker back to indifference. However, as the initial level of $\gamma$ is increased, risk aversion becoming more intense, the required compensatory increase in log ability becomes greater.

The figure is drawn on the assumption that the configuration of parameter values is such that both formal wage employment and self employment are each preferred by someone. The condition underlying this assumption is given in the following lemma.

Lemma 1. $W_{F}$ is always non-empty; $W_{S}$ is non-empty if and only if $w_{F}<\bar{\rho}(1-\psi)$.

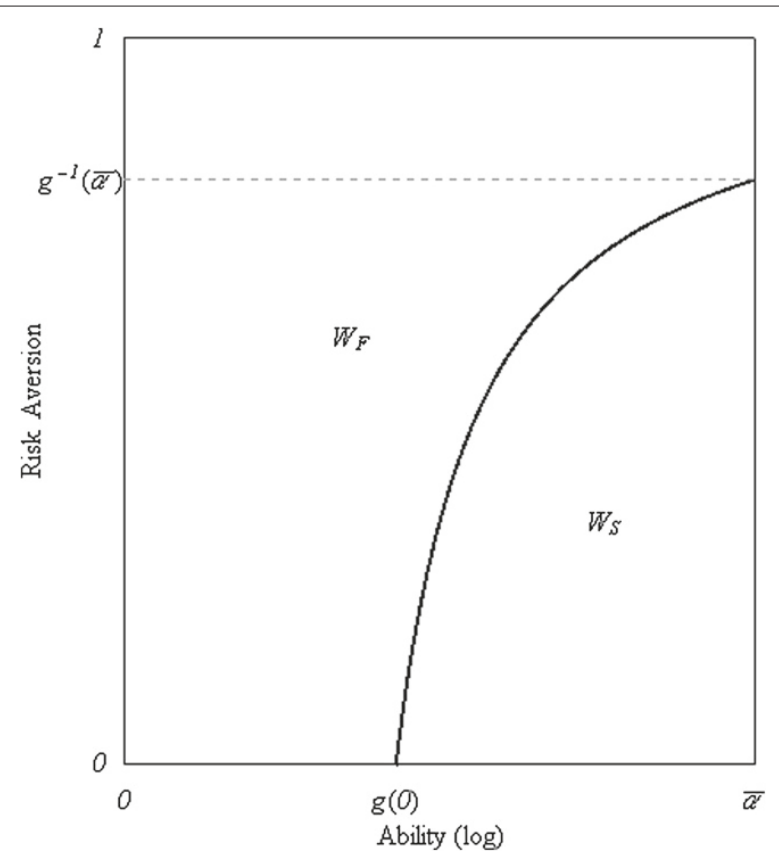

Figure 1 First choice sets. 
Intuitively, $W_{S}$ is non-empty if the formal wage rate $w_{F}$ is not too large. To explain why $W_{F}$ is non-empty, we note that the worker in the top left-hand corner of Figure 1 will always choose formal employment for any $w_{F}>0$. To see this, note that for this worker we have $v_{S}=(1-\psi) u(1)$, and hence $v_{F} / v_{S}=\left(w_{F}\right)^{1-\gamma} /(1-\psi)$. As $\gamma \longrightarrow 1$ we must have $v_{F}>v_{S}$, for $v_{F} / v_{S}$ approaches $1 /(1-\psi)>1$ for any positive value of $w_{F}$.

Before analyzing how the labour market operates in the presence of a fixed wage $w_{F}$, we specify the condition under which $w_{F}$ exceeds the wage $w_{F}^{*}$ that would clear the market with all first preferences being attained. From (3), $w_{F}^{*}$ is given by

$$
w_{F}^{*}=A_{F}-\left|W_{F}\left(w_{F}^{*}\right)\right| .
$$

To ensure that $w_{F}>w_{F}^{*}$, we must limit the demand intercept for formal labour, relative to the fixed formal wage rate $w_{F}$. Thus, we assume that the following condition holds:

Lemma 2. If $A_{F}<\left|W_{F}\left(w_{F}\right)\right|+w_{F}$ then there exists a unique wage $w_{F}^{*} \in\left(0, w_{F}\right)$ such that $L_{F}^{d}=\left|W_{F}\right|$.

Given that $w_{F}>w_{F}^{*}$, there is an excess supply of formal labour. To determine the equilibrium that then obtains we must specify a rationing scheme for formal jobs. Although we have assumed that $(\log )$ ability $\alpha$ only has a direct effect on income in self employment, all formal employees having the same income $w_{F}$, it may be supposed that some of the qualities that can make a worker successful in self employment, such as the ability to acquire relevant information and use initiative, may be conducive to seeking and obtaining jobs in a tight formal labour market. We therefore assume that the rationing scheme operates on the basis of ability: among those whose first preference is formal employment, the jobs go to those with the highest ability.

Writing a superscript $r$ to denote that this rationing scheme is in operation, the set of formal workers is then given by

$$
W_{F}^{r}: v_{F}>\max \left(v_{I}, v_{S}\right) \text { and } \alpha>\widehat{\alpha},
$$

where $\widehat{\alpha}$ satisfies

$$
A_{F}-w_{F}=\left|W_{F}^{r}(\widehat{\alpha})\right|
$$

and

$$
\left|W_{F}^{r}(\widehat{\alpha})\right|= \begin{cases}\int_{0}^{1} \int_{\widehat{\alpha}}^{\min (g(\gamma), \bar{\alpha})} f(\alpha, \gamma) \mathrm{d} \alpha \mathrm{d} \gamma & \text { if } \widehat{\alpha}<g(0) ; \\ \int_{g^{-1}(\widehat{\alpha})}^{1} \int_{\widehat{\alpha}}^{\min (g(\gamma), \bar{\alpha})} f(\alpha, \gamma) \mathrm{d} \alpha \mathrm{d} \gamma & \text { if } \widehat{\alpha}>g(0) .\end{cases}
$$

Note that if we were to allow $w_{F}=w_{F}^{*}$, then $\widehat{\alpha}=0$; but by asserting that $w_{F}>w_{F}^{*}$, it must be that $\widehat{\alpha}>0$. For a worker to be a member of the set $W_{F}^{r}$ it is necessary that formal employment be their first preference and that their (log) ability is above the critical level $\widehat{\alpha}$ that is required for success in the formal job market. $\widehat{\alpha}$ is determined so as to satisfy formal labour demand at wage $w_{F}$. The first line of (12) applies if the critical value $\widehat{\alpha}<g(0)$, the value of $\alpha$ at which a risk-neutral worker would be indifferent between $F$ and $S$. In this case workers with $\alpha>\widehat{\alpha}$ who are risk averse would also prefer $F$ to $S$. If, however, $\widehat{\alpha}>g(0)$, it must also be taken into account that workers for whom $\alpha>\widehat{\alpha}$, but 
whose level of risk aversion $\gamma$ is below the critical level $g^{-1}(\widehat{\alpha})$, would choose $S$ rather than $F$. This is represented in the second line of (12).

\subsubsection{Second preferences}

Those workers who are rationed out of formal employment choose between informal employment and self employment. According to their choices between these two alternatives, we define the respective sets

$$
\begin{aligned}
& W_{I}^{i}: v_{F}>v_{I}>v_{S} \text { and } \alpha<\widehat{\alpha} ; \\
& W_{S}^{i}: v_{F}>v_{S}>v_{I} \text { and } \alpha<\widehat{\alpha} .
\end{aligned}
$$

We denote the set $W_{S}^{i}$ as 'involuntarily' self employed, to distinguish it from the set $W_{S}$ defined in (7), which we shall henceforth refer to as the 'voluntarily' self employed. The set $W_{I}^{i}$ of workers in informal wage employment is also 'involuntary', but, for brevity we omit this term because all informal wage workers in the model are involuntary.

The borderline values of parameters underlying (13) and (14) are given by

$$
v_{S} \gtrless v_{I} \Leftrightarrow \alpha \gtrless h(\gamma),
$$

where

$$
h(\gamma)=\log w_{I}-\frac{\log (1-\psi)-\log (1-\phi)}{1-\gamma} .
$$

Given the rationing scheme, we then obtain the proportions of the labour force in sets $W_{I}^{i}$ and $W_{S}^{i}$ :

$$
\begin{array}{ll}
\left|W_{S}^{i}\right| & = \begin{cases}\int_{0}^{h^{-1}(\widehat{\alpha})} \int_{\max (0, h(\gamma))}^{\min (g(\gamma), \widehat{\alpha})} f(\alpha, \gamma) \mathrm{d} \alpha \mathrm{d} \gamma & \text { if } \widehat{\alpha}>h(0) ; \\
0 & \text { if } \widehat{\alpha}<h(0) ;\end{cases} \\
\left|W_{I}^{i}\right| & =1-\left|W_{S}\right|-\left|W_{F}^{r}\right|-\left|W_{S}^{i}\right| .
\end{array}
$$

Of workers for whom $\alpha<\widehat{\alpha}$, those with a combination of relatively high (log) ability and low risk aversion belong to set $W_{S}^{i}$, choosing involuntary self-employment; but if the value of $\alpha$ at the borderline between the two options here is greater than $\widehat{\alpha}$ then there is no involuntary self employment. The set of workers $W_{I}^{i}$ who choose informal wage employment follows as a residual.

For a given value of the endogenously determined wage rate $w_{I}$, the preferences of a worker with any characteristics $(\alpha, \gamma)$ are shown in our first proposition.

Proposition 1. Consider worker $i_{\alpha \gamma}$ with characteristics $(\alpha, \gamma)$. Then $i_{\alpha \gamma} \in W_{F}^{r}$ if $\alpha<$ $g(\gamma)$ and $\alpha>\widehat{\alpha} ; i_{\alpha \gamma} \in W_{S}$ if $\alpha>g(\gamma) ; i_{\alpha \gamma} \in W_{I}^{i}$ if $\alpha<h(\gamma)$ and $\alpha<\widehat{\alpha}$; and $i_{\alpha \gamma} \in W_{S}^{i}$ if $\alpha>h(\gamma), \alpha<g(\gamma)$ and $\alpha<\widehat{\alpha}$.

The preferences are illustrated in Figure 2. Here, it is assumed that Lemma 1 holds, so that $W_{S}$ is non-empty, and that the following lemma holds, to ensure that the other sets shown are non-empty. ${ }^{7}$

Lemma 3. $W_{I}^{i}$ is always non-empty; $W_{F}^{r}$ is non-empty if $\widehat{\alpha}<\bar{\alpha}$; $W_{S}^{i}$ is non-empty if $h(0)<\widehat{\alpha}$. 


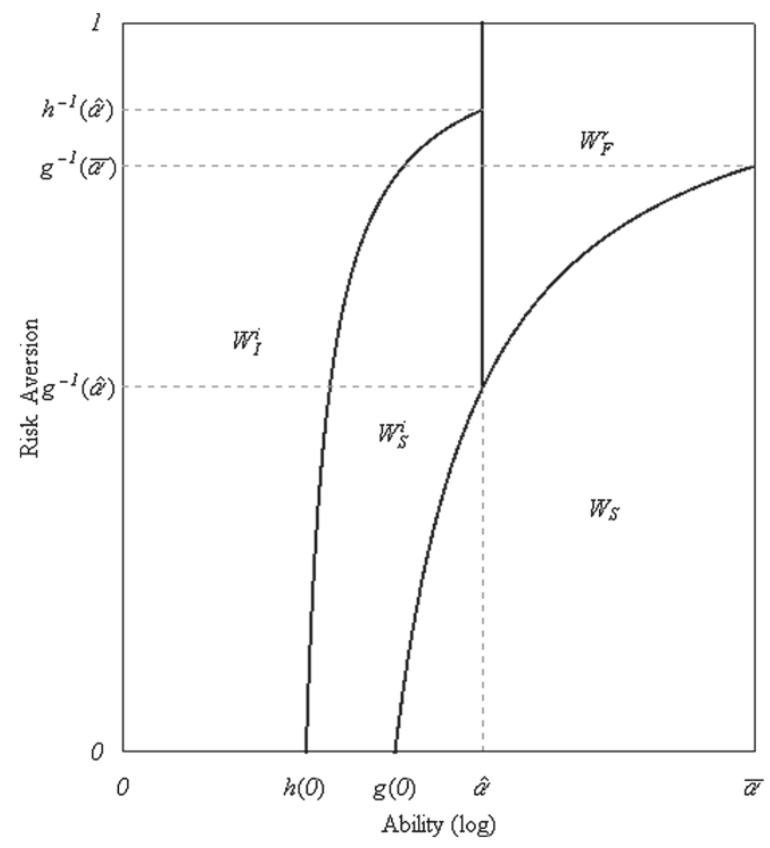

Figure 2 Labour supply allocation.

Workers with log ability greater than $\widehat{\alpha}$ divide between self employment and formal wage employment in a manner similar to that shown in Figure 1, and the explanation of the slope and curvature of the line dividing $W_{S}$ from $W_{F}^{r}$ is the same as that given with respect to Figure 1 . However, workers with log ability less than $\widehat{\alpha}$ do not obtain formal employment. Of this group, those with a combination of relatively low risk aversion and relatively high ability may choose self employment voluntarily. The remaining workers are rationed out of formal wage employment. Their preferences between involuntary self employment and informal wage employment reflect the same trade-offs and have the same intuitive explanation as we have already discussed in terms of Figure 1 for first preferences between self employment and formal wage employment. Hence, the explanation of why $W_{I}^{i}$ is non-empty is analogous to the earlier explanation of why $W_{F}$ is non-empty. Workers with sufficiently low ability may get informal wage jobs irrespective of their risk attitude; but those with a combination of sufficiently low risk aversion and high ability may become (involuntary) self employed.

\section{Equilibrium}

We now examine the equilibrium in the model. We begin by specifying a condition on parameter values for which, given that $w_{F}>w_{F}^{*}$, a market-clearing informal wage $w_{I}=$ $w_{I}^{*}$ below the formal wage $w_{F}$ exists.

Proposition 2. If $A_{I}<\left(1-\phi_{d}\right) w_{F}+\left|W_{I}^{i}\left(w_{F}\right)\right|$ then there exists a unique wage $w_{I}^{*} \in$ $\left(0, w_{F}\right)$ such that $L_{I}^{d}=\left|W_{I}^{i}\right|$.

The intercept on the informal labour demand curve must not be too high - for otherwise the condition that $w_{I}<w_{F}$ would be violated. Similarly, the condition imposes 
a lower bound on $w_{F}$, while the probability $\phi_{d}$ that an informal employer will renege on paying the wage must not be too high.

Given that the condition in Proposition 2 holds, we can interpret the wage $w_{I}$ underlying Figure 2 as the equilibrium wage $w_{I}^{*}$, so that the figure represents the equilibrium. The comparative statics of the model, to which we now turn, can be understood intuitively by reference to the figure. We are primarily interested in the effect of variations of parameter values on the size and membership of the sets shown in the figure; but most of these effects work through their impacts on two endogenous variables, the cut-off value $\alpha=\widehat{\alpha}$ of log ability and the informal wage rate $w_{I}$. We therefore begin by specifying the comparative statics of these variables.

The parameters $w_{F}, \phi_{c}$ and $\phi_{d}$ may be interpreted as, at least to some extent, policy variables. $w_{F}$ may be interpreted as a minimum wage rate imposed by the government. $\phi_{c}$ is the probability that informal wages will be confiscated by the government, and this can be increased by greater effort in detection or a greater willingness to confiscate once detected. $\phi_{d}$ is the probability that the employer will choose not to pay the informal wage. The government may be able to reduce this by strengthening contract enforcement of wage payment to informal workers. The parameters $A_{F}$ and $\bar{\alpha}$ may be interpreted as indicators of economic development. $A_{F}$ is the intercept of the demand curve for formal wage labour, and we may assume that the demand for the goods produced by formal wage labour increases as the economy grows. $\bar{\alpha}$ is the upper bound of log ability. An increase in $\bar{\alpha}$ can be interpreted as a rise in the average level of ability in the economy. The remaining parameters, $\psi$ and $A_{I}$ are less easily interpreted as policy tools or indicators of development.

Lemma 4. In equilibrium, the comparative statics of the log ability cut-off $\widehat{\alpha}$ and the informal wage rate $w_{I}$ are given by

Log ability cut-off

$$
\frac{d \widehat{\alpha}}{d A_{F}}<0 ; \quad \frac{d \widehat{\alpha}}{d w_{F}}>0 ; \quad \frac{d \widehat{\alpha}}{d \psi}>0 ; \quad \frac{d \widehat{\alpha}}{d \bar{\alpha}} \gtrless 0 ; \quad \frac{d \widehat{\alpha}}{d A_{I}}=\frac{d \widehat{\alpha}}{d \phi}=0 ;
$$

Informal wage rate

$$
\begin{aligned}
& \frac{d w_{I}}{d A_{F}}>0 ; \quad \frac{d w_{I}}{d w_{F}}<0 ; \quad \frac{d w_{I}}{d \psi}<0 ; \quad \frac{d w_{I}}{d \bar{\alpha}} \gtrless 0 ; \\
& \frac{d w_{I}}{d A_{I}} \quad>0 ; \quad \frac{d w_{I}}{d \phi_{d}}>\frac{d w_{I}}{d \phi_{c}}>0 .
\end{aligned}
$$

A higher demand for formal wage labour, as represented by $A_{F}$, is associated with a lower cut-off $\log$ ability $\widehat{\alpha}$, while a higher formal wage rate $w_{F}$ is associated with a higher cut-off ability. A higher value of $\psi$ represents a greater risk in self employment. At the margin, this causes the first preference of some voluntarily self employed workers to change to formal employment. The existence of relatively high ability among this group pushes up the cut-off value $\widehat{\alpha}$ at which the available formal jobs are filled. The demand for informal wage workers and income risk in informal wage employment have no impact on the formal employment decision. Although we shall be able to say something below about the effects of varying $\bar{\alpha}$, we are not able to sign $d \widehat{\alpha} / d \bar{\alpha}$ (or $\left.d w_{I} / d \bar{\alpha}\right)$. 
A higher demand for formal wage labour exerts upward pressure on the informal wage, whereas a higher formal wage has the opposite effect on the informal wage because it reduces formal labour demand. Greater risk in self employment causes a substitution effect from involuntary self employment to informal wage employment, with a negative impact on $w_{I}$. A higher demand for informal labour is associated with a higher wage $w_{I}$. Greater risk in informal wage employment causes a substitution of informal wage workers into involuntary self employment, exerting a positive impact on $w_{I}$. However, this impact is greater for $\phi_{d}$ than for $\phi_{c}$ : a marginal increase in the risk that the employer will renege on payment has a greater effect than the same marginal increase in the risk that the government will prevent both the worker and the employer from receiving informal income. Both of these increases in risk make informal wage employment less attractive to the worker, reducing informal labour supply and therefore increasing $w_{I}$. But, as we have explained, the latter effect has no effect on labour demand, whereas the former increases labour demand, and so pushes $w_{I}$ up further.

Using Lemma 4 and a standard stability condition on the relative slopes of labour demand and supply, we obtain the comparative statics of employment.

Proposition 3. In a stable equilibrium, the comparative statics of employment in each occupation are:

Voluntary self employment

$$
\begin{aligned}
& \frac{d\left|W_{S}\right|}{d \psi}<0 ; \quad \frac{d\left|W_{S}\right|}{d \bar{\alpha}}>0 ; \quad \frac{d\left|W_{S}\right|}{d w_{F}}<0 ; \\
& \frac{d\left|W_{S}\right|}{d A_{F}}=\frac{d\left|W_{S}\right|}{d A_{I}}=\frac{d\left|W_{S}\right|}{d \phi}=0 ;
\end{aligned}
$$

Formal wage employment

$$
\begin{aligned}
& \frac{d\left|W_{F}^{r}\right|}{d A_{F}}=1 ; \quad \frac{d\left|W_{F}^{r}\right|}{d w_{F}}=-1 ; \\
& \frac{d\left|W_{S}\right|}{d A_{F}}=\frac{d\left|W_{S}\right|}{d A_{I}}=\frac{d\left|W_{S}\right|}{d \phi}=\frac{d\left|W_{S}\right|}{d \psi}=\frac{d\left|W_{S}\right|}{d \bar{\alpha}}=0 ;
\end{aligned}
$$

Informal wage employment

$$
\begin{aligned}
& \frac{d\left|W_{I}^{i}\right|}{d A_{F}}<0 ; \quad \frac{d\left|W_{I}^{i}\right|}{d A_{I}}>0 ; \quad \frac{d\left|W_{I}^{i}\right|}{d \phi_{c}}<0 ; \\
& \frac{d\left|W_{I}^{i}\right|}{d \phi_{d}}>0 ; \quad \frac{d\left|W_{I}^{i}\right|}{d \psi}>0 ; \\
& \frac{d\left|W_{I}^{i}\right|}{d \bar{\alpha}} \gtrless 0 \Leftrightarrow \frac{d w_{I}}{d \bar{\alpha}} \lessgtr 0 ; \quad \frac{d\left|W_{I}^{i}\right|}{d w_{F}}>0 ;
\end{aligned}
$$

Involuntary self employment

$$
\begin{aligned}
& \frac{d\left|W_{S}^{i}\right|}{d A_{F}} \quad \gtrless 0 \Leftrightarrow \frac{d\left|W_{I}^{i}\right|}{d A_{F}} \lessgtr-1 ; \quad \frac{d\left|W_{S}^{i}\right|}{d A_{I}}<0 ; \quad \frac{d\left|W_{S}^{i}\right|}{d \phi_{c}}>0 ; \\
& \frac{d\left|W_{S}^{i}\right|}{d \phi_{d}} \quad<0 ; \quad \frac{d\left|W_{S}^{i}\right|}{d \psi} \gtrless 0 \Leftrightarrow\left|\frac{d\left|W_{S}\right|}{d \psi}\right| \gtrless \frac{d\left|W_{I}^{i}\right|}{d \psi} ; \quad \frac{d\left|W_{S}^{i}\right|}{d \bar{\alpha}} \gtrless 0 ; \\
& \frac{d\left|W_{S}^{i}\right|}{d w_{F}} \quad \gtrless 0 \Leftrightarrow \frac{d\left|W_{I}^{i}\right|}{d w_{F}} \lessgtr 1-\frac{d\left|W_{S}\right|}{d w_{F}} .
\end{aligned}
$$


As we might expect, voluntary self employment $\left|W_{S}\right|$ is decreasing in the riskiness $\psi$ of self employment income and in the formal wage rate $w_{F}$, but increasing in the average ability level in the labour force, as represented by $\bar{\alpha}$, while formal wage employment $\left|W_{F}^{r}\right|$ is increasing in the demand for formal labour, as given by the intercept $A_{F}$, and decreasing in $w_{F}$. More interesting results are obtained, however, for the other two employment types.

Informal wage employment $\left|W_{I}^{i}\right|$ is decreasing in the demand for formal labour, and it is increasing in the demand for informal wage labour and in the riskiness $\psi$ of self employment. Also, because a higher $w_{F}$ reduces $\left|W_{F}^{r}\right|,\left|W_{I}^{i}\right|$ is increasing in $w_{F}$. However, greater risk $\psi$ of not being paid an informal wage can affect $\left|W_{I}^{i}\right|$ in either direction, depending on the source of the risk. A higher risk $\phi_{c}$ that the government will confiscate the informal earnings of both the employer and employees in an informal firm results in a smaller $\left|W_{I}^{i}\right|$. But when the higher risk is in the form $\phi_{d}$ - that an employer is more likely to renege on payment - a greater $\left|W_{I}^{i}\right|$ results. This is because an increase in $\phi_{d}$ both depresses the informal wage, and increases the demand for informal wage labour by making it more elastic. The two effects conflict, but, assuming a stable market equilibrium in the informal labour market, the latter effect dominates. This suggests that the strengthening of detection by the authorities of informal wage work, but improvement of the contractual rights of informal workers to be paid their wages, would lead to a reduction in the amount of informal wage work. Finally, a higher level of average ability, as given by $\bar{\alpha}$, affects $\left|W_{I}^{i}\right|$ with the opposite sign to its effect on the informal wage $w_{I}$ - but this effect can go in either direction. Thus, an economy that is more developed in this sense may have more informal wage work. It is possible to show that $d w_{i} / d \bar{\alpha}$ is positive for sufficiently large $\bar{\alpha}$ and negative for $\bar{\alpha}$ sufficiently small. So, for small $\bar{\alpha}$, informal wage employment is increasing in the average level of ability, but this relationship reverses at higher levels of $\bar{\alpha}$.

Although involuntary self employment $\left|W_{S}^{i}\right|$ is found to be decreasing in the demand for informal wage labour, as represented by $A_{I}$, it may be increasing or decreasing in the demand for formal labour, $A_{F}$. This is because a rise in $A_{F}$ reduces the proportion of workers who do not achieve their first choice, but also depresses $w_{I}$. If the latter effect is sufficiently small, involuntary self employment is increasing in $A_{F}$. Thus, if economic development is associated with a greater demand for the output of formal wage work, it may be found that it is also associated with more involuntary self employment. Similarly, the effect on $\left|W_{S}^{i}\right|$ of a higher $w_{F}$ can be of either sign. Also, the effects on $\left|W_{S}^{i}\right|$ of greater risk are not clear-cut. Greater risk $\psi$ with respect to the earnings of the self employed can affect $\left|W_{S}^{i}\right|$ in either direction: there is a direct effect, discouraging involuntary self employment, but there is also an indirect effect which, by discouraging voluntary self employment, increases the supply of workers to involuntary self employment (and informal wage employment). The net effect can go either way, the underlying condition being that specified in the proposition. For similar reasons, a higher level of average ability, as represented by $\bar{\alpha}$ can affect $\left|W_{S}^{i}\right|$ in either direction. The effect on $\left|W_{S}^{i}\right|$ of an increase in the risks, $\phi_{c}$ and $\phi_{d}$, associated with informal wage employment is the opposite in sign to the effect of these risks on informal wage employment. But, since $d\left|W_{S}\right| / d \phi=d\left|W_{F}^{r}\right| / d \phi=0$, variation of $\left|W_{S}^{i}\right|$ in this case is exactly compensated by variation of the opposite sign in $\left|W_{I}^{i}\right|$. 


\section{Conclusions}

We formulate a model of an urban labour market in a developing economy in which workers' risk attitudes play a role in determining their labour supply decisions and we illustrate the model diagrammatically. Whereas in the Rauch (1991) model risk is excluded, with choices being made purely on the basis of ability, in our formulation trade-offs between ability and risk aversion matter. For example, when risk is excluded from the analysis a worker's first preference might be self employment (maximizing expected income); but, incorporating risk into the model, given that self employment income is uncertain, the worker's risk aversion may be sufficient to make him or her prefer formal wage employment. Similarly, the results of the search-and-matching literature on informality would be modified if risk aversion were taken into account. For example, in the model of Albrecht et al. (2009) workers who are relatively productive in formal-sector employment reject informal job offers to wait for a formal-sector job. But if risk aversion were allowed for, these workers might not take the risk of rejecting the informal job offer. Parallel to our analysis, it is therefore possible that a worker with higher ability would take an informal job, while a worker with lower ability would get a formal job.

Among our results is that a greater risk that an informal wage worker will not be paid can affect total informal wage employment in either direction, depending on the source of the risk. It is found that if the government wishes to reduce the amount of informal wage work, it may do so by strengthening detection efforts by the authorities of informal wage work, or by improving the contractual rights of informal wage workers to be paid. The latter policy discourages informal employers from offering so many jobs. But this policy would have no effect on formal wage employment or voluntary self employment - the reduction in informal wage employment would be exactly compensated by an increase in involuntary self employment. We also find that if the average level of ability is used as a development indicator, informal wage employment at first rises and then falls as the economy develops. If, alternatively, the demand for formal production (and thus labour) is used as a development indicator, development can be associated with more involuntary self employment.

Our analysis indicates some relatively complex relationships between risk attitudes and labour allocation. In Figure 2 it can be seen that, among the voluntary self employed, higher ability is, on average, associated with greater risk aversion. The same observation can be made for the involuntarily self employed. However, if we consider the whole group of self employed - voluntary plus involuntary - the monotonic relationship disappears. The ability level at which risk aversion is, on average, smallest, is at the cut-off level of ability for formal wage employment. Also, looking horizontally up the diagram, it can be seen that, for relatively low and relatively high levels of risk aversion, movements between types of employment are monotonic as ability rises. However, at an intermediate level of risk aversion, increasing ability is associated with a shift from informal wage employment to (involuntary) self employment and on to wage employment, but then back to self employment (which is voluntary in this case). This is consistent with the findings of Perry et al. (2007), who observe young workers often take informal wage employment to develop their skills and may move into formal wage work, but eventually utilize their skills in voluntary self employment.

Our formulation might be developed in various directions, including a full modelling of the demand side of the labour market. This might include consideration of whether 
the outputs of the informal and formal wage sectors are substitutable. Allowance might also be made for ability to affect labour productivity in formal wage jobs, and it would be interesting to examine how the results are affected if the rationing scheme for formal wage jobs were changed, for example by supposing that jobs are allocated among applicants randomly.

\section{Appendix}

Lemma 1. First consider a worker with characteristics $(\rho, \gamma)=(1,1-\varepsilon)$ where $\varepsilon>0$. Then, since $w_{F}>0, v_{F} / v_{S}=\left(w_{F}\right)^{\varepsilon} /(1-\psi) \longrightarrow 1 /(1-\psi)$ as $\varepsilon \longrightarrow 0$. Therefore, as $\psi>0$, we have that $1 /(1-\psi)>1$. Hence, by continuity, there exists an $\varepsilon$ sufficiently close to zero such that $v_{F} / v_{S}>1$, so that $W_{F} \neq \varnothing$. Second, consider the circumstances under which $v_{F}<v_{S}$ for an agent with characteristics $(\rho, \gamma) . v_{F}<v_{S}$ if and only if $\left(w_{F}\right)^{1-\gamma /(1-\gamma)<}$ $(1-\psi) \rho^{1-\gamma} /(1-\gamma) \Longleftrightarrow w_{F} / \rho<(1-\psi)^{1 / 1-\gamma}<0$. The function $w_{F} / \rho$ is decreasing in $\rho$ and, since $1-\psi<1$, the function $(1-\psi)^{1 / 1-\gamma}$ is decreasing in $\gamma$. Therefore if $w_{F} / \bar{\rho}>1-\psi$ it must be that $w_{F} / \rho>(1-\psi)^{1 / 1-\gamma}$ for all $1<\rho<\bar{\rho}$ and $0<\gamma<1$; i.e., $W_{S}=\varnothing$. Thus, $W_{S} \neq \varnothing$ if and only if $w_{F}<\bar{\rho}(1-\psi)$.

Lemma 2. Let $w$ denote a candidate wage in the formal sector. First suppose that $w=0$; then $L_{F}^{d}=A_{F}>0$. It follows that $\left|W_{F}\right|=0$ since $v_{S}>v_{F}=0$. Hence $L_{F}^{d}>\left|W_{F}\right|$ if $w=0$. Next suppose that $w=w_{F}$, in which case $L_{F}^{d}=A_{F}-w_{F}$. So if $A_{F}<\left|W_{F}\left(w_{F}\right)\right|+w_{F}$ then $L_{F}^{d}<\left|W_{F}\right|$. Hence, by the intermediate value theorem, there must exist $w_{F}^{*} \in\left(0, w_{F}\right)$ such that $L_{F}^{d}=\left|W_{F}\right|$. Since $L_{F}^{d}$ is linear and $\frac{\partial\left|W_{F}\right|}{\partial w_{F}}=\frac{1}{\bar{\alpha}} \int_{\zeta}^{g^{-1}(\bar{\alpha})} \frac{\partial g}{\partial w_{F}} d \gamma>0$ (where $\zeta=0$ if $g(0) \geqslant 0$ and $g^{-1}(0)$ if $\left.g(0)<0\right) w_{F}^{*}$ is unique.

Proposition 1. First note that if $w_{F}>w_{I}$ then $g(\gamma)>h(\gamma)$ for all $\gamma \in[0,1)$ and $v_{F}>v_{I}$. Firstly consider under what conditions a worker will enter formal employment, i.e. $i_{\alpha \gamma} \in$ $W_{F}^{r} \Longleftrightarrow v_{F}>\max \left(v_{I}, v_{S}\right)$ and $\alpha>\widehat{\alpha}$. This is satisfied if and only if $\alpha<g(\gamma)$ and $\alpha>\widehat{\alpha}$. Next consider when self-employment would be a worker's first choice, i.e. $i_{\alpha \gamma} \in W_{S} \Longleftrightarrow$ $v_{S}>\max \left(v_{F}, v_{I}\right) \Longleftrightarrow \alpha>g(\gamma)$. The condition $\alpha>g(\gamma)$ is sufficient, for the condition $\alpha>h(\gamma)$ that guarantees $v_{S}>v_{I}$ is implied by $\alpha>g(\gamma)$. Now consider a worker's second choice if they are unable to attain their first preference of formal employment. The conditions for a worker to enter informal employment are $i_{\alpha \gamma} \in W_{I}^{i} \Longleftrightarrow \alpha<h(\gamma)$ and $\alpha<\widehat{\alpha}$. This is true since $v_{I}>v_{S} \Longleftrightarrow \alpha<h(\gamma)$. The conditions for a worker to enter self-employment are $i_{\alpha \gamma} \in W_{S}^{i} \Longleftrightarrow v_{F}>v_{S}>v_{I}$ and $\alpha<\widehat{\alpha}$. This holds if and only if $h(\gamma)<\alpha<g(\gamma)$ and $\alpha<\widehat{\alpha}$.

Lemma 3. Consider a worker $i_{\rho \gamma}$ with characteristics $(\rho, \gamma)=(1,1-\varepsilon)$ where $\varepsilon>0$. We now find the conditions under which $i_{\rho \gamma} \in W_{I}^{i}$ using the inequalities in (13). First, from (2), $v_{F}>v_{I} \Longleftrightarrow\left(w_{F}\right)^{\varepsilon}>(1-\phi)\left(w_{I}\right)^{\varepsilon}$. Then, since $\lim _{\varepsilon} \longrightarrow 0\left(w_{F}\right)^{\varepsilon}=1$ and $\lim _{\varepsilon \longrightarrow 0}(1-\phi)\left(w_{I}\right)^{\varepsilon}=(1-\phi)$, there must exist $\varepsilon_{1}$ such that $v_{F}>v_{I}$ for all $\varepsilon<\varepsilon_{1}$. Second, using (2) we have $v_{I}>v_{S} \Longleftrightarrow(1-\phi)\left(w_{I}\right)^{\varepsilon}>(1-\psi)$. Since $\psi>\phi$ then there will exist $\varepsilon_{2}$ such that for all $\varepsilon<\varepsilon_{2}, v_{I}>v_{S}$. Therefore, since $0<\widehat{\alpha}, i_{\rho \gamma} \in W_{I}^{i}$ for $\varepsilon<\min \left(\varepsilon_{1}, \varepsilon_{2}\right)$, i.e. $W_{I}^{i} \neq \varnothing$. Next consider a worker with characteristics $(\rho, \gamma)=(\widehat{\rho}, 1-\varepsilon)$ where $\varepsilon>0$. As above, $v_{F}>v_{I}$. Then, since $\lim _{\varepsilon \longrightarrow 0}\left(w_{F}\right)^{\varepsilon}=\lim _{\varepsilon \rightarrow 0}(\widehat{\rho})^{\varepsilon}=1$ and $(1-\psi)<1$, there must exist $\varepsilon>0$ such that $v_{F}>v_{S}$. Hence, if $\widehat{\alpha}<\bar{\alpha}$, then $i_{\rho \gamma} \in W_{F}^{r}$ and $W_{F}^{r} \neq \varnothing$. Last, consider a worker with characteristics $(\rho, \gamma)=(\widehat{\rho}, 1-\varepsilon)$, where $\widehat{\rho}=e^{\widehat{\alpha}}$. Then 
there exists $\varepsilon>0$ such that $(1-\phi)\left(w_{I}\right)^{\varepsilon}>(1-\psi) \widehat{\rho}^{\varepsilon}$, i.e., such that $v_{I}>v_{S}$. For a worker with $(\rho, \gamma)=(\widehat{\rho}, 0)$ then $v_{S}>v_{I} \Longleftrightarrow w_{I}(1-\phi)<\widehat{\rho}(1-\psi)$. Therefore, if $w_{I}(1-\phi)<\widehat{\rho}(1-\psi)$, which is equivalent to $h(0)<\widehat{\alpha}$, by the intermediate value theorem, there must exist $0<\tilde{\gamma}<1$ such that $h(\widetilde{\gamma})=\widehat{\alpha}$. We know that $h(\gamma)<g(\gamma)$ and $v_{F}>v_{S} \Longleftrightarrow \alpha<g(\gamma)$, hence $\widehat{\alpha}=h(\tilde{\gamma})<g(\widetilde{\gamma})$. Therefore, there must exist $\eta, \mu>0$ such that a worker with $(\rho, \gamma)=(\widehat{\rho}-\eta, \tilde{\gamma}-\mu)$ satisfies $v_{F}>v_{S}>v_{I}$. That is $W_{S}^{i} \neq \varnothing$.

Proposition 2. Let $w$ denote a candidate informal wage. First suppose that $w=0$, then $L_{I}^{d}=A_{I}>0 .\left|W_{I}^{i}\right|=0$ since $v_{I}=0$ and $v_{S}>0$. Hence $L_{I}^{d}>\left|W_{I}^{i}\right|$. Next, suppose that $w=w_{F}$, in which case $L_{I}^{d}=A_{I}-\left(1-\phi_{d}\right) w_{F}$. So, if $A_{I}<\left(1-\phi_{d}\right) w_{F}+\left|W_{I}^{i}\left(w_{F}\right)\right|$, then $L_{I}^{d}<\left|W_{I}^{i}\right|$ for $w=w_{F}$. Therefore, by the intermediate value theorem, there must exist $w_{I}^{*} \in\left(0, w_{F}\right)$ such that $L_{I}^{d}=\left|W_{I}^{i}\right|$. Since $L_{I}^{d}$ is linear and $\frac{\partial\left|W_{I}^{i}\right|}{\partial w_{F}}=\frac{1}{\bar{\alpha}} \int_{\zeta}^{g^{-1}(\widehat{\alpha})} \frac{\partial h}{\partial w_{I}} d \gamma>0$ (where $\zeta=0$ if $h(0) \geqslant 0$ and $h^{-1}(0)$ if $\left.h(0)<0\right)$ the equilibrium is unique.

Lemma 4. From (9) the derivatives of $g(\cdot)$ satisfy $g_{\psi}>0$ and $g_{w_{F}}>0$, with the remaining derivatives being zero. The derivatives of $g^{-1}(\cdot)$ satisfy $g_{w_{F}}^{-1}<0, g_{\alpha}^{-1}>0$, and $g_{\psi}^{-1} \gtrless 0 \Leftrightarrow \log w_{F} \gtrless \alpha$. As we encounter $g_{\psi}^{-1}$ only when $\log w_{F}<\alpha$, we have $g_{\psi}^{-1}<0$. Using these results to differentiate $\left|W_{F}^{r}(\widehat{\alpha})\right|$ in (12) we obtain

$$
\begin{aligned}
& \frac{\partial\left|W_{F}^{r}(\widehat{\alpha})\right|}{\partial \widehat{\alpha}}= \begin{cases}\frac{g^{-1}(\widehat{\alpha})-1}{\bar{\alpha}}<0 & \text { if } \widehat{\alpha}>g(0) ; \\
-\frac{1}{\bar{\alpha}}<0 & \text { if } \widehat{\alpha}<g(0) ;\end{cases} \\
& \frac{\partial\left|W_{F}^{r}(\widehat{\alpha})\right|}{\partial \bar{\alpha}}= \begin{cases}-\frac{1}{\bar{\alpha}^{2}} \int_{g^{-1}}^{g^{-1}(\bar{\alpha})} g(\gamma) \mathrm{d} \gamma-\frac{\widehat{\alpha}}{\bar{\alpha}^{2}} g^{-1}(\widehat{\alpha})+\frac{\widehat{\alpha}}{\bar{\alpha}^{2}} \gtrless 0 & \text { if } \widehat{\alpha}>g(0) ; \\
-\frac{1}{\bar{\alpha}^{2}} \int_{0}^{g^{-1}(\bar{\alpha})} g(\gamma) \mathrm{d} \gamma+\frac{\widehat{\widehat{\alpha}}}{\bar{\alpha}^{2}} \gtrless 0 & \text { if } \widehat{\alpha}<g(0) ;\end{cases} \\
& \frac{\partial\left|W_{F}^{r}(\widehat{\alpha})\right|}{\partial \psi}= \begin{cases}\frac{1}{\bar{\alpha} w_{F}}\left(g^{-1}(\bar{\alpha})-g^{-1}(\widehat{\alpha})\right)>0 & \text { if } \widehat{\alpha}>g(0) ; \\
\frac{g^{-1}(\bar{\alpha})}{\bar{\alpha} w_{F}}>0 & \text { if } \widehat{\alpha}<g(0) ;\end{cases} \\
& \frac{\partial\left|W_{F}^{r}(\widehat{\alpha})\right|}{\partial w_{F}}=\left\{\begin{array}{ll}
\frac{1}{\bar{\alpha} w_{F}}\left(g^{-1}(\bar{\alpha})-g^{-1}(\widehat{\alpha})\right)>0 & \text { if } \widehat{\alpha}>g(0) ; \\
\frac{g^{-1}(\bar{\alpha})}{\bar{\alpha} w_{F}}>0 &
\end{array}\right] \\
& \frac{\partial\left|W_{F}^{r}(\widehat{\alpha})\right|}{\partial A_{F}}=\frac{\partial\left|W_{F}^{r}(\widehat{\alpha})\right|}{\partial A_{I}}=\frac{\partial\left|W_{F}^{r}(\widehat{\alpha})\right|}{\partial \phi}=0 .
\end{aligned}
$$

Hence, total differentiation of (11) yields

$$
\begin{aligned}
\frac{d \widehat{\alpha}}{d A_{F}} & =\left(\frac{\partial\left|W_{F}^{r}(\widehat{\alpha})\right|}{\partial \widehat{\alpha}}\right)^{-1}<0 ; \\
\frac{d \widehat{\alpha}}{d \psi} & =-\frac{\partial\left|W_{F}^{r}(\widehat{\alpha})\right|}{\partial \psi}\left(\frac{\partial\left|W_{F}^{r}(\widehat{\alpha})\right|}{\partial \widehat{\alpha}}\right)^{-1}>0 ; \\
\frac{d \widehat{\alpha}}{d w_{F}} & =-\left(1+\frac{\partial\left|W_{F}^{r}(\widehat{\alpha})\right|}{\partial w_{F}}\right)\left(\frac{\partial\left|W_{F}^{r}(\widehat{\alpha})\right|}{\partial \widehat{\alpha}}\right)^{-1}>0 ; \\
\frac{d \widehat{\alpha}}{d \bar{\alpha}} & =-\frac{\partial\left|W_{F}^{r}(\widehat{\alpha})\right|}{\partial \bar{\alpha}}\left(\frac{\partial\left|W_{F}^{r}(\widehat{\alpha})\right|}{\partial \widehat{\alpha}}\right)^{-1} \gtrless 0 ; \\
\frac{d \widehat{\alpha}}{d A_{I}} & =\frac{d \widehat{\alpha}}{d \phi}=0 .
\end{aligned}
$$


We proceed in the same manner for the comparative statics of $w_{I}$. We have $h_{\phi}<0$, $h_{\psi}>0$ and $h_{\phi}^{-1}>0, h_{\psi}^{-1}<0$. Differentiation of $\left|W_{I}^{i}\right|$ yields:

$$
\begin{aligned}
& \frac{\partial\left|W_{I}^{i}\right|}{\partial A_{F}}=\frac{1}{\bar{\alpha}} \frac{\partial \widehat{\alpha}}{\partial A_{F}}\left(1-h^{-1}(\widehat{\alpha})\right)<0 ; \\
& \frac{\partial\left|W_{I}^{i}\right|}{\partial \phi}=\left\{\begin{array}{l}
-\frac{1}{\bar{\alpha}} \int_{0}^{h^{-1}(\widehat{\alpha})} \frac{\partial h}{\partial \phi} \mathrm{d} \gamma<0 \text { if } h(0) \geqslant 0 ; \\
-\frac{1}{\bar{\alpha}} \int_{h^{-1}(0)}^{h^{-1}(\widehat{\alpha})} \frac{\partial h}{\partial \phi} \mathrm{d} \gamma<0 \text { if } h(0)<0 ;
\end{array}\right. \\
& \frac{\partial\left|W_{I}^{i}\right|}{\partial \psi}=\left\{\begin{array}{l}
\frac{1}{\bar{\alpha}} \int_{0}^{h^{-1}(\widehat{\alpha})} \frac{\partial h}{\partial \psi} \mathrm{d} \gamma+\frac{1}{\bar{\alpha}} \frac{\partial \widehat{\alpha}}{\partial \psi}\left(1-h^{-1}(\widehat{\alpha})\right)>0 \text { if } h(0) \geqslant 0 ; \\
\frac{1}{\bar{\alpha}} \int_{h^{-1}(0)}^{h^{-1}(\widehat{\alpha})} \frac{\partial h}{\partial \psi} \mathrm{d} \gamma+\frac{1}{\bar{\alpha}} \frac{\partial \widehat{\alpha}}{\partial \psi}\left(1-h^{-1}(\widehat{\alpha})\right)>0 \text { if } h(0)<0 ;
\end{array}\right. \\
& \frac{\partial\left|W_{I}^{i}\right|}{\partial \bar{\alpha}}=\left\{\begin{array}{l}
\frac{-1}{\bar{\alpha}^{2}} \int_{0}^{h^{-1}(\widehat{\alpha})} h(\gamma) \mathrm{d} \gamma+\left(\frac{1}{\bar{\alpha}} \frac{\partial \widehat{\alpha}}{\partial \bar{\alpha}}-\frac{\widehat{\alpha}}{\bar{\alpha}^{2}}\right)\left(1-h^{-1}(\widehat{\alpha})\right) \gtrless 0 \text { if } h(0) \geqslant 0 ; \\
\frac{-1}{\bar{\alpha}^{2}} \int_{h^{-1}(0)}^{h^{-1}(\widehat{\alpha})} h(\gamma) \mathrm{d} \gamma+\left(\frac{1}{\bar{\alpha}} \frac{\partial \widehat{\alpha}}{\partial \bar{\alpha}}-\frac{\widehat{\alpha}}{\bar{\alpha}^{2}}\right)\left(1-h^{-1}(\widehat{\alpha})\right) \gtrless 0 \text { if } h(0)<0 ;
\end{array}\right. \\
& \frac{\partial\left|W_{I}^{i}\right|}{\partial w_{F}}=\frac{1}{\bar{\alpha}} \frac{\partial \widehat{\alpha}}{\partial w_{F}}\left(1-h^{-1}(\widehat{\alpha})\right)>0 ;
\end{aligned}
$$

Hence, total differentiation of the expression $\left|W_{I}^{i}\right|-L_{I}^{d}=0$ yields

$$
\begin{aligned}
& \frac{d w_{I}}{d A_{F}}=-\left(\frac{\partial\left|W_{I}^{i}\right|}{\partial A_{F}}\right) /\left(\frac{\partial\left|W_{I}^{i}\right|}{\partial w_{I}}-\frac{\partial L_{I}^{d}}{\partial w_{I}}\right)>0 ; \\
& \frac{d w_{I}}{d A_{I}}=-\left(\frac{\partial\left|W_{I}^{i}\right|}{\partial A_{I}}-1\right) /\left(\frac{\partial\left|W_{I}^{i}\right|}{\partial w_{I}}-\frac{\partial L_{I}^{d}}{\partial w_{I}}\right)>0 ; \\
& \frac{d w_{I}}{d \phi_{c}}=-\left(\frac{\partial\left|W_{I}^{i}\right|}{\partial \phi_{c}}\right) /\left(\frac{\partial\left|W_{I}^{i}\right|}{\partial w_{I}}-\frac{\partial L_{I}^{d}}{\partial w_{I}}\right)>0 ; \\
& \frac{d w_{I}}{d \phi_{d}}=\frac{d w_{I}}{d \phi_{c}}+\left\{w_{I} /\left(\frac{\partial\left|W_{I}^{i}\right|}{\partial w_{I}}-\frac{\partial L_{I}^{d}}{\partial w_{I}}\right)\right\}>\frac{d w_{I}}{d \phi_{c}} ; \\
& \frac{d w_{I}}{d \psi}=-\left(\frac{\partial\left|W_{I}^{i}\right|}{\partial \psi}\right) /\left(\frac{\partial\left|W_{I}^{i}\right|}{\partial w_{I}}-\frac{\partial L_{I}^{d}}{\partial w_{I}}\right)<0 ; \\
& \frac{d w_{I}}{d \bar{\alpha}}=-\left(\frac{\partial\left|W_{I}^{i}\right|}{\partial \bar{\alpha}}\right) /\left(\frac{\partial\left|W_{I}^{i}\right|}{\partial w_{I}}-\frac{\partial L_{I}^{d}}{\partial w_{I}}\right) \gtrless 0 ; \\
& \frac{d w_{I}}{d w_{F}}=-\left(\frac{\partial\left|W_{I}^{i}\right|}{\partial w_{F}}\right) /\left(\frac{\partial\left|W_{I}^{i}\right|}{\partial w_{I}}-\frac{\partial L_{I}^{d}}{\partial w_{I}}\right)<0 .
\end{aligned}
$$


Proposition 3. Using (10), the comparative statics of $\left|W_{S}\right|$ are determined as:

$$
\begin{aligned}
& \frac{d\left|W_{S}\right|}{d \psi}=\left\{\begin{array}{l}
-\frac{1}{\bar{\alpha}} \int_{0}^{g^{-1}(\bar{\alpha})} \frac{\partial g(\gamma)}{\partial \psi} \mathrm{d} \gamma<0 \text { if } g(0) \geqslant 0 ; \\
-\frac{1}{\bar{\alpha}} \int_{g^{-1}(0)}^{g^{-1}(\bar{\alpha})} \frac{\partial g(\gamma)}{\partial \psi} \mathrm{d} \gamma<0 \text { if } g(0)<0 ;
\end{array}\right. \\
& \frac{d\left|W_{S}\right|}{d \bar{\alpha}}=\left\{\begin{array}{l}
\frac{1}{\bar{\alpha}^{2}} \int_{0}^{g^{-1}(\bar{\alpha})} g(\gamma) \mathrm{d} \gamma>0 \text { if } g(0) \geqslant 0 ; \\
\frac{1}{\bar{\alpha}^{2}} \int_{g^{-1}(0)}^{g^{-1}(\bar{\alpha})} g(\gamma) \mathrm{d} \gamma>0 \text { if } g(0)<0 ;
\end{array}\right. \\
& \frac{d\left|W_{S}\right|}{d W_{F}}=\left\{\begin{array}{l}
-\frac{1}{\bar{\alpha}} \int_{0}^{g^{-1}(\bar{\alpha})} \frac{\partial g(\gamma)}{\partial \psi} \mathrm{d} \gamma<0 \text { if } g(0) \geqslant 0 ; \\
-\frac{1}{\bar{\alpha}} \int_{g^{-1}(0)}^{g^{-1}(\bar{\alpha})} \frac{\partial g(\gamma)}{\partial \psi} \mathrm{d} \gamma<0 \text { if } g(0)<0 ;
\end{array}\right. \\
& \frac{d\left|W_{S}\right|}{d A_{F}}=\frac{d\left|W_{S}\right|}{d A_{I}}=\frac{d|W S|}{d \phi}=0 .
\end{aligned}
$$

The comparative statics for $\left|W_{F}^{r}\right|$ are determined trivially from differentiation of the right side of (11). The comparative statics of $\left|W_{I}^{i}\right|$ are determined by differentiating informal labour demand in (4). We then obtain

$$
\begin{array}{rlrl}
\frac{d\left|W_{I}^{i}\right|}{d A_{F}} & =-\left(1-\phi_{d}\right) \frac{d w_{I}}{d A_{F}}<0 ; & \frac{d\left|W_{I}^{i}\right|}{d A_{I}}=1-\left(1-\phi_{d}\right) \frac{d w_{I}}{d A_{I}}<0 ; \\
\frac{d\left|W_{I}^{i}\right|}{d \phi_{c}} & =-\left(1-\phi_{d}\right) \frac{d w_{I}}{d \phi_{c}}<0 ; & \frac{d\left|W_{I}^{i}\right|}{d \phi_{d}}=-\left(1-\phi_{d}\right) \frac{d w_{I}}{d \phi_{d}}+w_{I}>0 ; \\
\frac{d\left|W_{I}^{i}\right|}{d \psi} & =-\left(1-\phi_{d}\right) \frac{d w_{I}}{d \psi}>0 ; & \frac{d\left|W_{I}^{i}\right|}{d \bar{\alpha}}=-\left(1-\phi_{d}\right) \frac{d w_{I}}{d \bar{\alpha}} \gtrless 0 \Leftrightarrow \frac{d w_{I}}{d \bar{\alpha}} \lessgtr 0 ; \\
\frac{d\left|W_{I}^{i}\right|}{d w_{F}} & =-\left(1-\phi_{d}\right) \frac{d w_{I}}{d w_{F}}>0 ;
\end{array}
$$

where stability of the equilibrium in the informal labour market, i.e. $d\left|W_{I}^{i}\right| / d w_{I}>$ $d L_{I}^{d} / d w_{I}$, implies $d w_{I} / d A_{I}<1-\phi_{d}$ and $d w_{I} / d \phi_{d}<w_{I} /\left(1-\phi_{d}\right)$. Last, we obtain the comparative statics of $\left|W_{S}\right|$ by noting that, for any exogenous variable $x$, it must hold that $d\left|W_{I}^{i}\right| / d x=-\left(d\left|W_{S}\right| / d x+d\left|W_{I}^{i}\right| / d x+d\left|W_{F}^{r}\right| / d x\right)$.

\section{Endnotes}

${ }^{1}$ Fortin et al. (1997) is an exception: the Rauch-Lucas model is adapted to include a probability of less than unity of finding a formal job and workers are assumed risk averse. Specific results are generated by incorporating this formulation into a CGE model for Senegal. See Bennett (2011) for a recent contribution and references to literature that develops Rauch's formulation.

${ }^{2}$ Whereas we assume that informal employers may renege on paying the endogenouslydetermined informal wage, Basu et al. (2010) analyse the case of possible non-compliance by (formal) employers with a minimum wage law. In their analysis the government chooses the intensity of enforcement, and so compliance decisions by heterogenous employers are made endogenously. Our analysis is simpler in that the probability that an 
employer will renege is assumed the same for all workers and is given exogenously. However, the Basu et al. model does not allow for risk aversion.

${ }^{3}$ See Kanbur (2009) on the meanings and definition of informality.

${ }^{4}$ A recent paper by Bennett and Rablen (2012) uses a similar approach to model voluntary and involuntary labour market states, also incorporating endogenous labour demand, but no allowance is made for risk.

${ }^{5}$ We choose a simple formulation of the utility function for tractability. Other simple specifications such as constant absolute risk aversion or mean-variance utility can instead be used and yield similar results. However, the assumption of constant relative risk aversion has stronger empirical support (Wakker 2008).

${ }^{6}$ The case shown here is illustrative. It is also feasible that this line will start at a point on the $\gamma$-axis (though it will still have the slope and curvature shown).

${ }^{7}$ Lemmas 1 and 3 specify restrictions on parameter values so that each of the employment states that the model covers obtain simultaneously, as is observed in developing economies. Without Lemma 3, for example, the return to self employment might be so high that neither type of wage labour would exist in equilibrium.

\section{Competing interests}

The IZA Journal of Labor \& Development is committed to the IZA Guiding Principles of Research Integrity. The authors declare that they observed these principles.

\section{Acknowledgements}

We are grateful to Hartmut Lehmann and an anonymous referee for very helpful comments.

Responsible editor: Hartmut Lehmann

\section{Author details \\ ${ }^{1}$ Department of Economics and Finance, and CEDI, Brunel University, Uxbridge, Middlesex, UB8 3PH, UK. ${ }^{2}$ IZA, Bonn, Germany. ${ }^{3}$ Westminster Business School, University of Westminster, 35 Marylebone Road, London, NW1 5LS, UK.}

Received: 25 November 2012 Accepted: 14 December 2012

Published: 28 December 2012

\section{References}

Albrecht J, Navarro L, Vroman S (2009) The effects of labour market policies in an economy with an informal sector. Econ J 119: 1105-1129

Basu AK, Chau NH, Kanbur R (2010) Turning a blind eye: Costly enforcement, credible commitment and minimum wage laws. Econ J 120: 244-269

Bennett J (2011) Informal production and labour market segmentation. J Inst Theor Econ 167: 686-707

Bennett J, Rablen MD (2012) Self-employment, wage employment and informality in a developing economy. IZA Discussion Paper No. 6406

Deshingkar P, Akter S (2009) Migration and human development in India, UNDP Human Development Research Paper 2009/13

Fortin B, Marceau N, Savard L (1997) Taxation, wage controls and the informal sector. J Pub Econ 66: 293-312

Günther I, Launov A (2012) Informal employment in developing economies: Opportunity or last resort? J Dev Econ 97: $88-98$

Kanbur R (2009) Conceptualizing informality: Regulation and enforcement. Cornell University

Lucas RE (1978) On the size distribution of business firms. Bell J Econ Manag Sci 9: 508-523

OECD (2009) Is informal normal? Towards more and better jobs in developing countries. OECD, Paris

Perry GE, Maloney WF, Arias OS, Fajnzylber P, Mason AD, Saavedra-Chanduvi J (2007) Informality: Exit and exclusion. World Bank, Washington

Rauch JE (1991) Modelling the informal sector formally. J Dev Econ 35: 33-47

Wakker PP (2008) Explaining the characteristics of the power (crra) utility family. Health Econ 17: 1329-1344

World Bank (2012) The World Development Report 2012: Jobs. World Bank, Washington

Zenou Y (2008) Job search and mobility in developing countries. Theory and policy implications. J Dev Econ 86: 336-355

doi:10.1186/2193-9020-1-5

Cite this article as: Bennett et al:: Risk attitudes and informal employment in a developing economy. IZA Journal of Labor \& Development 2012 1:5. 